\title{
Forbush decreases and turbulence levels at coronal mass ejection fronts
} \author{
(The GRAPES-3 collaboration)

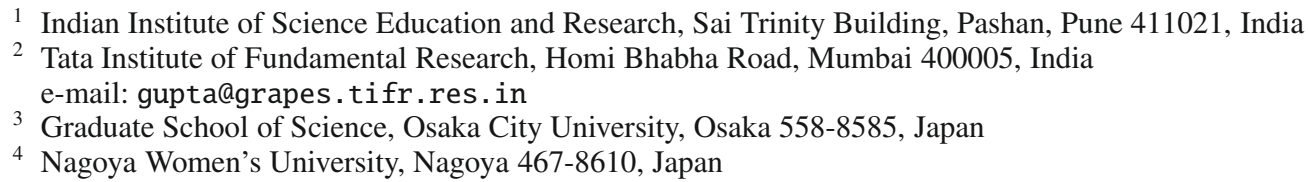

P. Subramanian ${ }^{1}$, H. M. Antia ${ }^{2}$, S. R. Dugad ${ }^{2}$, U. D. Goswami², S. K. Gupta², Y. Hayashi ${ }^{3}$, N. Ito $^{3}$, S. Kawakami ${ }^{3}$, H. Kojima ${ }^{4}$, P. K. Mohanty ${ }^{2}$, P. K. Nayak ${ }^{2}$, T. Nonaka ${ }^{3}$, A. Oshima ${ }^{3}$, K. Sivaprasad ${ }^{2}$, H. Tanaka ${ }^{2}$, and S. C. Tonwar ${ }^{2}$

Received 11 February 2008 / Accepted 25 October 2008

\section{ABSTRACT}

\begin{abstract}
Aims. We seek to estimate the average level of MHD turbulence near coronal mass ejection (CME) fronts as they propagate from the Sun to the Earth.

Methods. We examined the cosmic ray data from the GRAPES-3 tracking muon telescope at Ooty, together with the data from other sources for three closely observed Forbush decrease events. Each of these event is associated with frontside halo coronal mass ejections (CMEs) and near-Earth magnetic clouds. The associated Forbush decreases are therefore expected to have significant contributions from the cosmic-ray depressions inside the CMEs/ejecta. In each case, we estimate the magnitude of the Forbush decrease using a simple model for the diffusion of high-energy protons through the largely closed field lines enclosing the CME as it expands and propagates from the Sun to the Earth. The diffusion of high-energy protons is inhibited by the smooth, large-scale magnetic field enclosing the CME and aided by the turbulent fluctuations near the CME front. We use estimates of the cross-field diffusion coefficient $D_{\perp}$ derived from the published results of extensive Monte Carlo simulations of cosmic rays propagating through turbulent magnetic fields. We then compare our estimates with the magnitudes of the observed Forbush decreases.

Results. Our method helps constrain the ratio of energy density in the turbulent magnetic fields to that in the mean magnetic fields near the CME fronts. This ratio is found to be $\sim 2 \%$ for the 2001 April 11 Forbush decrease event, $\sim 6 \%$ for the 2003 November 20 Forbush decrease event and 249\% for the much more energetic event of 2003 October 29.
\end{abstract}

Key words. ISM: cosmic rays - Sun: coronal mass ejections (CMEs) - Sun: solar-terrestrial relations

\section{Introduction}

Forbush decreases are short-term depressions in the cosmic ray flux reaching the Earth, and they are caused by the effects of the interplanetary counterparts of coronal mass ejections (CMEs) from the Sun (and the shocks they drive) and also the corotating interaction regions originating from the Sun. They have been studied closely since their initial discovery in the 1930s (see, e.g., comprehensive observational reviews by Cane 2000; Venkatesan \& Badruddin 1990). With the recent upsurge of interest in space weather effects due to solar transients, the complementary information provided by the cosmic ray signatures of these effects has assumed increased significance.

In this work, we have examined the data from the GRAPES-3 tracking muon telescope at Ooty for three wellobserved events. Earlier, we had analysed the Forbush decrease data from this experiment during the period 2001-2004 and searched for events that can be associated with a nearEarth magnetic cloud and a corresponding halo CME from the Sun. We start from lists of magnetic cloud events given on the WIND webpage, http://lepmfi.gsfc.nasa.gov/mfi/ mag_cloud_pub1.html, Lynch et al. (2003) and Huttunen et al. (2005). We shortlist Forbush decrease events that occurred in a reasonable time window around the time of entry of the magnetic clouds. Of these shortlisted events, we have selected the three events shown in Fig. 8. Two of these events (2001 April 11 and 2003 October 29) have especially clean decrease profiles. Even though the decrease profile for the third event on 2003 November 20 is not nearly as clear-cut, we still selected it, because of its association with a very closely observed magnetic cloud, and also because it illustrates the diversity of the events observed with the GRAPES-3 experiment. We estimate the contribution of the depressed cosmic ray density inside the CME to the total Forbush decrease seen in these datasets, and use it to derive conclusions regarding the level of turbulence near the CME front.

The events discussed in this work were very closely observed, and they have their origins in full halo CMEs that originated close to the centre of the solar disc. This means that the corresponding interplanetary CMEs (ICMEs) would have been intercepted at the Earth as ejecta/magnetic clouds, and the observed cosmic ray depressions corresponding to these events would have had contributions from the shock ahead of the ICME, as well as from the ejecta/magnetic cloud itself (Cane et al. 1994, 1995; Cane \& Richardson 1997). Theoretical treatments of Forbush decreases model the effect as arising due to a general propagating region of enhanced turbulence/scattering 
and decreased diffusion (e.g., Nishida 1983; le Roux \& Potgieter 1991) and do not distinguish between the shock and the ejecta, or implicitly assume that the decrease is only due to the shock (e.g., Chih \& Lee 1986). It is fairly well known that magnetic clouds are well-correlated with Forbush decreases (e.g., Zhang \& Burlaga 1988; Badruddin et al. 1986; Badruddin et al. 1991; Venkatesan \& Badruddin 1990; Sanderson et al. 1990; see, however, Lockwood et al. 1991, for the opposite viewpoint).

The relationship between the decrease due to the shock and the one due to the magnetic cloud has been investigated (Cane et al. 1995, 1997; Wibberenz et al. 1997, 1998), and there is some evidence that they can contribute in roughly equal proportions, to the overall magnitude of the decrease, although the individual time profiles of the decrease due to these two effects can be quite different (Wibberenz et al. 1997). It is worth mentioning that the contributions of the shock and the magnetic cloud to the overall decrease can be discerned relatively easily from the spacecraft observations (e.g., Cane et al. 1995, 1997; Cane 2000), since the temporal boundaries of the magnetic cloud can be readily ascertained by using the data from the same source. These data typically exhibit two steps in the decrease phase, one corresponding to the shock passage and the second to the entry of the magnetic cloud. The two-step signature, however, need not be a necessary condition for the Forbush decreases arising from the combination of a magnetic cloud and the associated shock. Even for events where there is clear evidence that both the shock and the following magnetic cloud have intercepted the Earth (as is the case with the three events presented in this paper), the precise decrease profile will depend upon the standoff distance between the magnetic cloud and the shock and on the energies of the protons detected. If the protons are energetic enough that their mean free path is comparable to the magnetic cloud-shock standoff distance, the distinct identities of the steps corresponding to the shock and the cloud may not be clear. We attribute $50 \%$ of the total decrease to the magnetic cloud ( $\alpha=0.5$, see Sect. 3), following the general logic outlined by Wibberenz et al. (1997). We discuss the sensitivity of our final results to variations in $\alpha$ in Sect. 7. In our quantitative work, we estimate the magnitude of the Forbush decrease using filtered data (see Sect. 2.1 for a description of the filtering method) averaged over $1 \mathrm{~h}$.

The high muon counting rate measured by the GRAPES-3 experiment results in extremely small statistical errors, allowing small changes in the intensity of the cosmic ray flux to be measured with high precision. Thus a small drop $(\sim 0.2 \%)$ in the cosmic ray flux, during a Forbush decrease event can be reliably detected. This is possible even in the presence of the diurnal anisotropy of much larger magnitude $(\sim 1.0 \%)$, through a suitable filtering technique described subsequently.

Before we proceed further, it is worth discussing the terminology we use. The term CME is generally used to denote the blob of plasma ejected from the solar corona as viewed by coronographs near the Sun (typically from 1 to $30 R_{\odot}$ ). The CME can be thought of as a largely closed, magnetic flux rope-like magnetic structure (see, however, Bothmer et al. 1996, 1997, for evidence of CMEs possessing closed, as well as some open field lines) whose cross-section expands as it travels through the heliosphere. There are practically no high-energy cosmic rays inside it when it starts out from near the Sun, and the ambient cosmic rays diffuse into it via cross-field diffusion across the closed magnetic field lines as it travels through the heliosphere. The term "ICME" is a broad one used to denote the interplanetary counterpart of a CME (see, e.g., Forsyth et al. 2006; Wimmer-Schweingruber et al. 2006, and references therein), while the term "magnetic cloud" is reserved for ICMEs detected

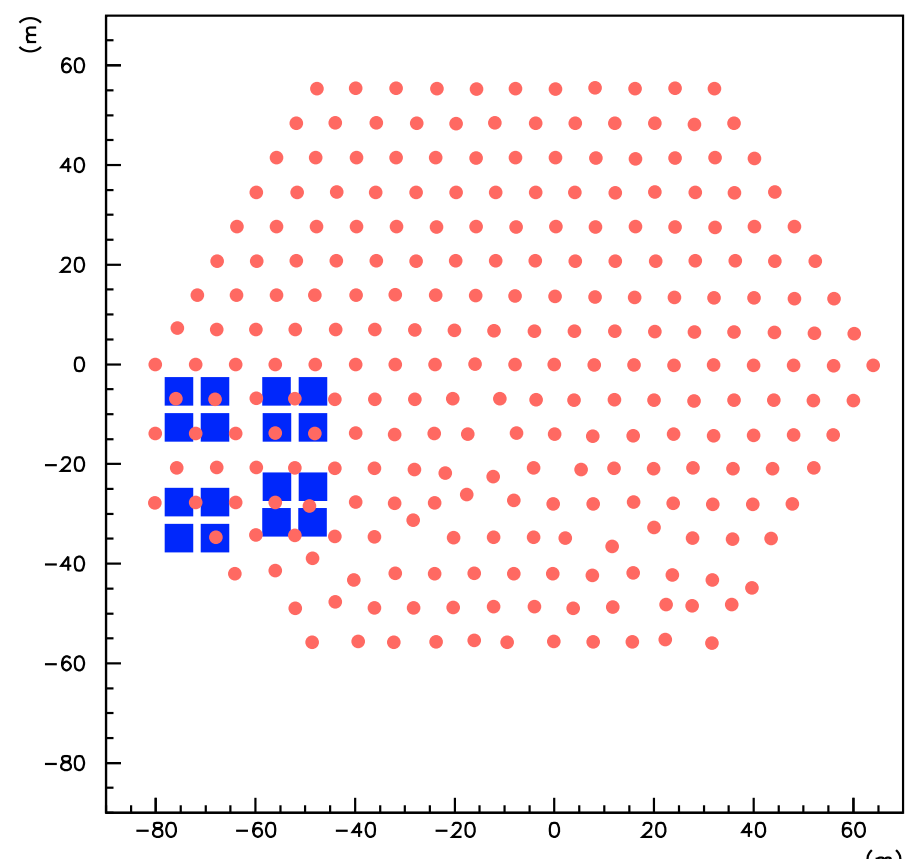

(m)

Fig. 1. A schematic layout the GRAPES-3 air shower array shown here with 217 detectors (circles). Each of the 16 squares represent a $35 \mathrm{~m}^{2}$ area tracking muon detector with $E_{\mu} \geq 1 \mathrm{GeV}$ used in the present work.

near the Earth, possessing certain well-defined criteria such as plasma temperature depressions and smooth magnetic field rotations (see, e.g., Burlaga et al. 1981; Bothmer \& Schwenn 1998). Other CME/ICME related ejecta detected near the Earth are merely called "ejecta".

The rest of this paper is organised as follows, We briefly describe the GRAPES-3 experiment in Sect. 2. We describe the model we used to calculate the magnitude of the expected Forbush decreases in Sect. 3. The three Forbush decrease events we analysed are described in Sects. 4-6. We describe our results in Sect. 7 and summarise them in Sect. 8.

\section{GRAPES-3 experimental system}

The GRAPES-3 experiment is located at Ooty $\left(11.4^{\circ} \mathrm{N}\right.$ latitude, $76.7^{\circ} \mathrm{E}$ longitude, and $2200 \mathrm{~m}$ altitude), in southern India. The GRAPES-3 air shower experiment was designed to have a compact configuration of a conventional type array, with a separation of only $8 \mathrm{~m}$ between the adjacent detectors, which are deployed in a symmetric hexagonal geometry. A schematic layout of the GRAPES-3 array is shown in Fig. 1. The observations were started in 2000 with 217 detectors, located within the inner 8 rings (see Fig. 1 Gupta et al. 2005).

A very large-area tracking muon telescope operating as a part of the GRAPES-3 experiment (Gupta et al. 2005; Hayashi et al. 2005), is a unique instrument, to search for the high-energy protons emitted during the active phase of a solar flare or a coronal mass ejection (CME). The muon telescope is capable of providing a high-statistics, directional study of muons. The GRAPES-3 muon telescope covers an area of $560 \mathrm{~m}^{2}$, consisting of a total of 16 modules, each $35 \mathrm{~m}^{2}$ in area. These modules are located close to each other as shown in Fig. 1. A cluster of four $35 \mathrm{~m}^{2}$ area neighbouring modules, located inside a common hall, constitutes one supermodule with a total area of $140 \mathrm{~m}^{2}$. The energy threshold of the telescope is $1 \mathrm{GeV}$ for the muons arriving 


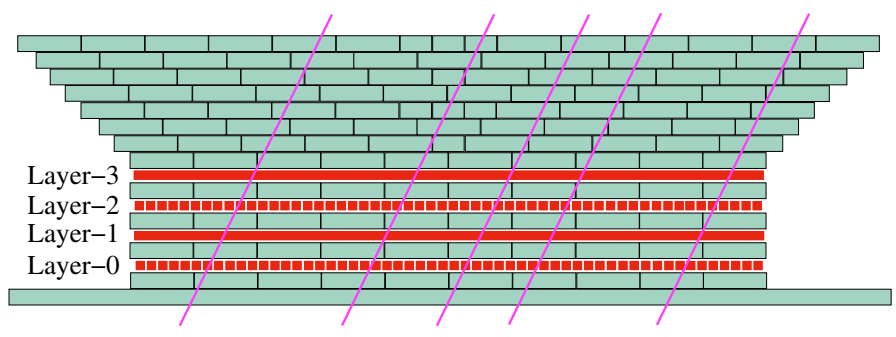

Fig. 2. A schematic display of the 4-layer tracking muon telescope module with 58 PRCs per layer. The four layers of the PRCs labelled Layer-0, Layer-1, etc. are embedded in concrete blocks. Inclined lines represent a set of parallel muon tracks.

along the vertical direction. The cutoff rigidity due to the magnetic field of the Earth at Ooty is $17 \mathrm{GV}$ in the vertical direction and varies from 12 to $42 \mathrm{GV}$ across the field of view of the muon telescope as shown later in Fig. 6.

The basic detector element of the muon telescope is a rugged proportional counter (PRC) made from a $600 \mathrm{~cm}$ long steel pipe with $2.3 \mathrm{~mm}$ wall thickness and a square cross-sectional area of $10 \times 10 \mathrm{~cm}^{2}$. A muon telescope module with a sensitive area of $35 \mathrm{~m}^{2}$ consists of a total of 232 PRCs arranged in four layers of 58 PRCs each, with alternate layers placed in orthogonal directions. Two successive layers of the PRCs are separated by a $15 \mathrm{~cm}$ thick concrete layer, consisting of $60 \times 60 \times 15 \mathrm{~cm}^{3}$ blocks as shown in Fig. 2. The four-layer PRC configuration of the muon modules allows a $3-\mathrm{D}$ reconstruction of the muon track direction to an accuracy of $\sim 6^{\circ}$. It is worth noting that the accuracy gradually increases with increasing zenith angle due to the greater separation of the triggered PRCs.

To achieve an energy threshold of $1 \mathrm{GeV}$ for vertical muons, an absorber of total thickness $\sim 550 \mathrm{~g} \mathrm{~cm}^{-2}$ in the form of concrete blocks is employed. This was done by placing a total of 15 layers of concrete blocks above Layer-1, as shown schematically in Fig. 2. A unique feature of the GRAPES-3 muon module is the robust structure of the PRCs, which supports the huge load of $2.4 \mathrm{~m}$ thick concrete in a self-supporting manner. The concrete blocks are arranged in the shape of an inverted pyramid to shield the PRCs, with coverage up to $45^{\circ}$ around the vertical direction for the incident muons. The threshold energy changes to $\sec \theta \mathrm{GeV}$ for the muons incident at a zenith angle of $\theta$. The cross section of a muon telescope module is shown schematically in Fig. 2. A cluster of four such modules, separated by a horizontal distance of $130 \mathrm{~cm}$ at the base constitutes one supermodule. The GRAPES-3 muon telescope contains a total of four supermodules (Hayashi et al. 2005).

Because of the sensitivity of the PRCs to low-energy $\gamma$ rays, from the radioactivity present in the concrete absorber, individual PRCs display sizable counting rates of $\sim 200 \mathrm{~Hz}$. An output is generated, if any one of the 58 PRCs produces a signal. A logical "OR" of outputs from all 58 PRCs in a layer is generated, after suitable amplification and shaping to form the layer OR output. A coincidence of the four OR outputs from the four layers in a module is used to generate the 4-layer trigger. Despite high counting rates of individual PRCs, the 4-layer coincidence trigger is relatively free of the contribution from the background radioactivity and it is caused only by the passage of a muon. The observed 4-layer muon counting rate of $\sim 3200 \mathrm{~Hz}$ per module yields a total counting rate $\sim 3 \times 10^{6} \mathrm{~min}^{-1}$ for all 16 modules. This high rate permits small changes of $\lesssim 0.1 \%$ in the muon flux, to be detected on a timescale of $\sim 5 \mathrm{~min}$, after appropriate correction to the variation in the atmospheric pressure with time.

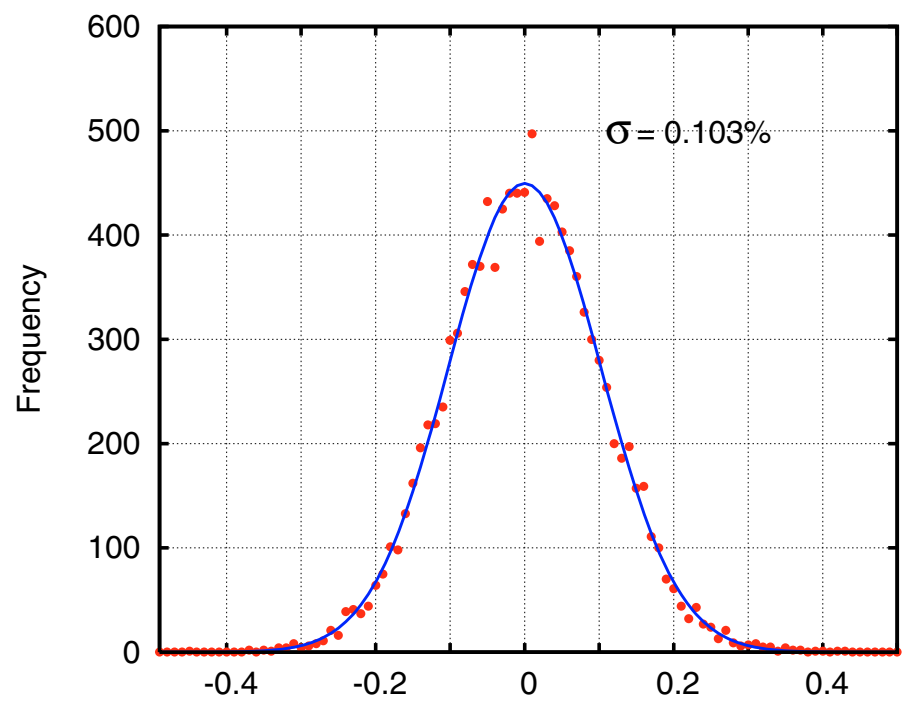

Fig. 3. Distribution of the relative variation in $\%$, for the 4-layer muon counting rate for the $35 \mathrm{~m}^{2}$ area modules for time intervals of $6 \mathrm{~min}$. Also shown is a Gaussian fit to the data. The standard deviation of the fit is $0.103 \%$ as against an expected value of $0.095 \%$ based on statistics.

Most of the detected muons are generated by $\gtrsim 20 \mathrm{GeV}$ galactic cosmic rays, and form a stable and dominant background to the variation in their flux produced by the CME/solar flare. The muon data is grouped online every $10 \mathrm{~s}$, into solidangle bins of $\sim 0.05 \mathrm{sr}$, consistent with the angular resolution of the muon telescope as described below. Since $>1 \mathrm{GeV}$ muons are secondaries produced by the primary protons of energy $\gtrsim 20 \mathrm{GeV}$ in the atmosphere, therefore these observations can be used to probe the effect of the Sun on cosmic rays.

The data recorded during 2003 October, from 15 out of 16 working modules were used in the statistical analysis described below. The muon rate for each $35 \mathrm{~m}^{2}$ area module was recorded for 770 time intervals, each 6 min in duration spread over a total of about 3.5 days. During this period of 3.5 days, the data could not be recorded for 70 out of 840 intervals, due to the failure of the recording system in the first supermodule. Next, the mean muon rate of the module for the entire duration was computed. Then the percent deviation from the mean module rate was calculated, separately for each module. Finally, the rms spread in the percent deviation called "relative variation" was calculated for each module for all 6-min intervals. In Fig. 3, the distribution of the relative variation in the 4-layer muon rate in percent, as recorded by the muon modules is shown. Also shown in Fig. 3, is a Gaussian fit to this distribution, which yields a standard deviation of $0.103 \%$. This value may be compared with an expectation of $0.095 \%$, based on a 6 -min muon statistic of $1.1 \times 10^{6}$ per module. This tiny statistical error in muon rate allows a high-precision study of various solar phenomena to be carried out (Nonaka et al. 2006).

\subsection{Data analysis}

In all four supermodules, the direction of muons is recorded into 225 solid-angle bins. This was done by using a dedicated direction-sensitive trigger with an independent data acquisition system for each of the four supermodules. As shown in Fig. 4, the muon angle is determined for each PRC in the lower layer and binned into 15 angular bins based on the specific location of the PRC triggered in the upper layer from among the 15 PRCs, one directly above (central PRC) and 7 each on either side of the 


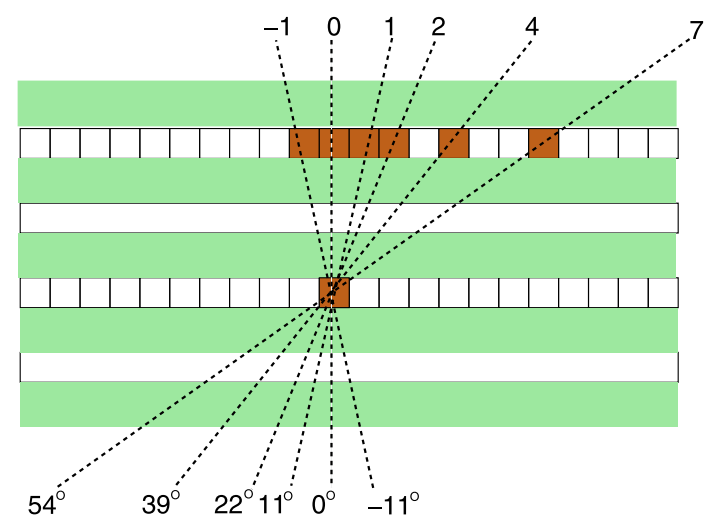

Fig. 4. A schematic view of muon arrival angle selection based on the PRC triggered in the lower and 15 PRCs in the upper layer. The triggered PRCs are shown as filled squares.

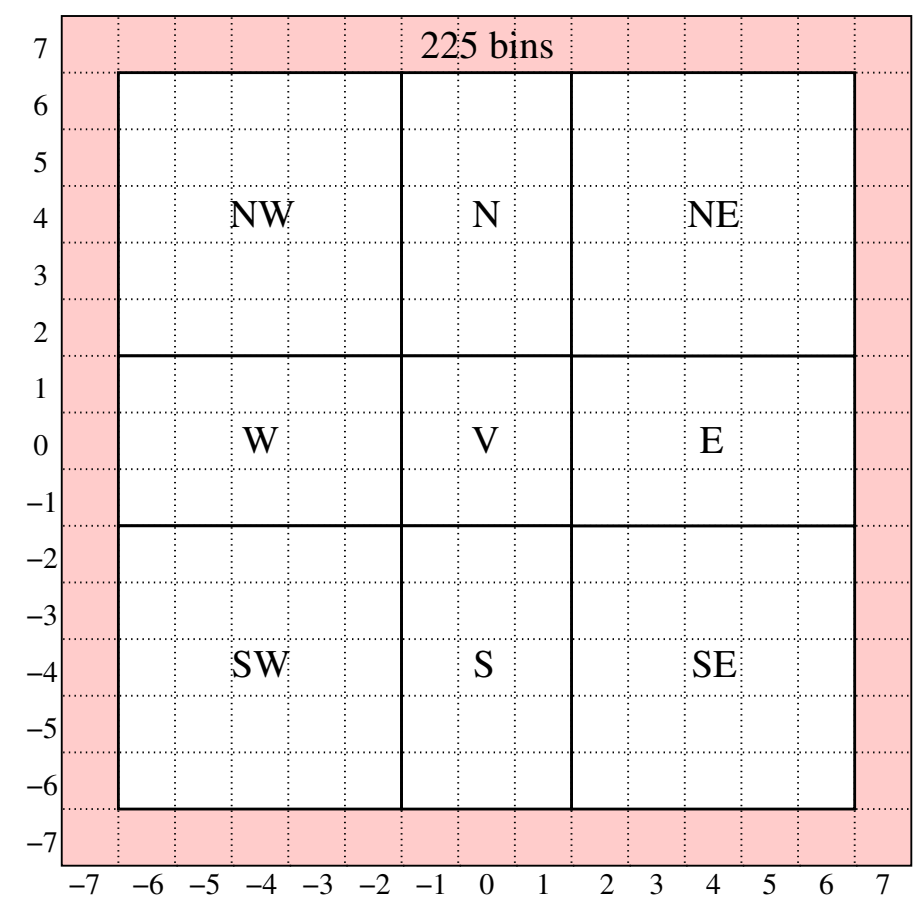

Fig. 5. A schematic of the nine muon arrival direction bins; $3 \times 3$ vertical bin $\mathrm{V}$, and four $3 \times 5$ central bins $\mathrm{N}, \mathrm{E}, \mathrm{W}, \mathrm{S}$, and four $5 \times 5$ outer bins NE, SE, SW, NW.

central PRC. This angular binning is carried out in each of the two orthogonal projections ( $X Z$ and $Y Z ; Z$ is vertical direction), thereby generating a 2 -dimensional $15 \times 15=225$ solid-angle map of muon directions. The contents of the 225 solid-angle bins are recorded, once every $10 \mathrm{~s}$, thus providing a continuous monitoring of the directional flux of muons in the sky.

The variation in the muon rate may be studied in any of the 225 solid angle bins. However, it is expected that the influence of a solar flare and/or CME would be spread over several bins. This directional spread could arise from the influence of the terrestrial, solar, and interplanetary magnetic fields, etc. Therefore, the detected muons have been regrouped into $3 \times 3=9$ coarse solid-angle bins, as shown schematically in Fig. 5. This regrouping of the data was done by combining either a set of $3 \times 5$ or $5 \times 5$ fine solid-angle bins. The exception being the vertical direction where $3 \times 3$ bins have been combined. This choice of angular segmentation was dictated by the fact that the muon flux is comparatively larger for the near central directions (N, E, W, S)

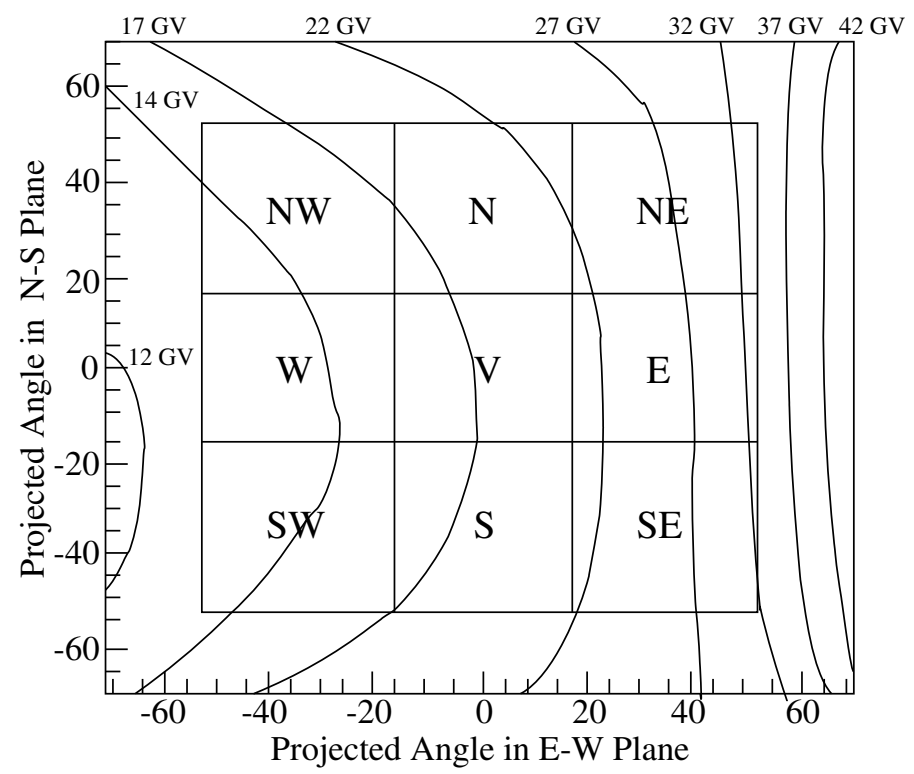

Fig. 6. The 9 coarse solid-angle bins are shown along with the contours of constant geomagnetic cutoff rigidity in the field of view (FOV). Cutoff rigidity varies from 12 to $42 \mathrm{GV}$ in the FOV of GRAPES-3.

than for the outer directions (NE, SE, NW, SW). This choice results in a relatively similar solid-angle coverage for the nine coarse bins. Thus the solid-angle of acceptance includes only $13 \times 13=169$ out of original 225 bins, restricting the maximum zenith angle to $50^{\circ}$. This still exceeds the shielding coverage of $45^{\circ}$, for the PRCs at the outer edge, but such events constitute $<1 \%$ of the data. This regrouping also results in muon statistics for various bins that are not too dissimilar.

At the energies $(\gtrsim 20 \mathrm{GeV})$ of interest here, the propagation of charged particles near the Earth $\left(<20 R_{\mathrm{E}}\right.$, where $R_{\mathrm{E}}$ is the radius of the Earth) is strongly influenced by the geomagnetic field. The access by a charged particle to a given geographical location depends on the momentum per unit charge of the particle called rigidity. The threshold value of the rigidity is termed "geomagnetic cutoff rigidity", which depends on the geographical location on the Earth and the direction of the arriving particle. The geomagnetic cutoff rigidity can be calculated using a detailed model of the geomagnetic field (Cooke et al. 1991). The geomagnetic cutoff rigidity for the field of view (FOV) of the GRAPES-3 muon telescope varies significantly for the nine coarse, solid-angle bins. We first calculate the cutoff rigidity for the centre of each of the 169 fine solid-angle bins, which constitute the 9 elements of the FOV, using the IGRF2000 geomagnetic field model. Subsequently, a weighted mean of the cutoff rigidities of the fine bins constituting a coarse bin is calculated for each of the 9 coarse bins. These weights are the muon counting rates for a given fine bin. Here, it needs to be emphasised that the knowledge of the geomagnetic field at any particular moment in time is imperfect, and it is virtually impossible to determine the cutoff to a high degree of accuracy. However, the calculated values of the geomagnetic cutoff represent a very useful approximation to the true values at the time of the observations. In Fig. 6 the contours of constant geomagnetic cutoff rigidity in the FOV are superimposed over a schematic of the 9 solid-angle bins of muon arrival directions. The geomagnetic cutoff rigidity varies from $12 \mathrm{GV}$ in the west to $42 \mathrm{GV}$ in the east, within the FOV of the GRAPES-3 tracking muon telescope.

In this study we used the GRAPES-3 data summed over a time interval of one hour for each of the nine bins, which are 


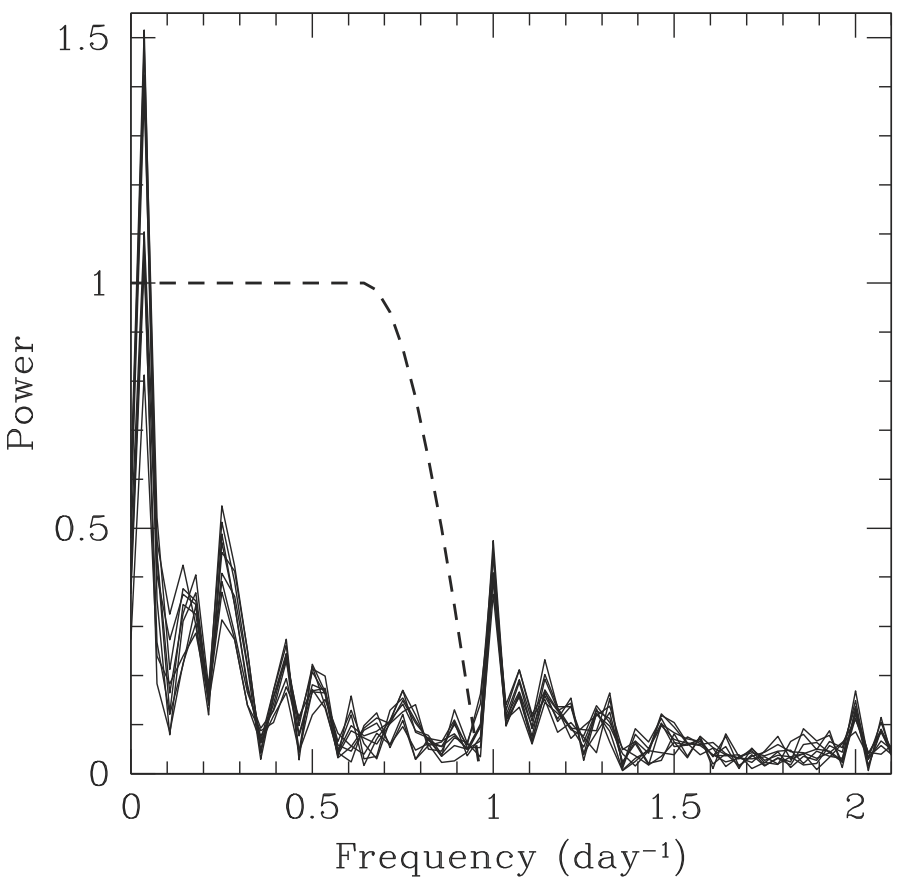

Fig. 7. The power spectrum of 28 day data covering the period from 26 March to 22 April 2001. The solid lines show the results for all 9 directions used in the study while the dashed line shows the function that is used for filtering out the high-frequency components. The Fourier transform is multiplied by this function before taking the inverse Fourier transform to get filtered time series.

identified as NW, N, NE, W, V, E, SW, S, and SE. The calculated cutoff rigidities for these nine bins are 15.5, 18.7, 24.0, $14.3,17.2,22.4,14.4,17.6$, and $22.4 \mathrm{GV}$, respectively, as seen from Fig. 6. The summing over an interval of $1 \mathrm{~h}$ improves the signal-to-noise ratio, but the diurnal variations in the muon flux are still present. To filter out these oscillations we applied a lowpass filter, removing all frequencies higher than 1 day $^{-1}$. In Fig. 7 we show the Fourier transform of the data covering a period of 28 days that includes the event of 2001 April 11. The peaks corresponding to diurnal variation and its first harmonic are visible. The Fourier transform is multiplied by the function shown by a dashed line to remove the high-frequency components. This filter is found to be effective in removing high-frequency oscillations, including the diurnal variations and their harmonics. The Forbush decrease events are also clearer in the filtered data.

The smoothing may tend to change the amplitude of the decrease and possibly shift the onset time for the Forbush decrease by a few hours in some cases. However, it is often difficult to determine if these differences are artifacts of smoothing or whether the unfiltered data showed different amplitude because a diurnal oscillation happened to have the right phase, so as to enhance or reduce the amplitude of the Forbush decrease. Some fluctuations in muon flux could be due to Forbush decrease and associated events, but it is unlikely that these will be periodic in nature, hence are not likely to be affected by the filter. Since the differences in amplitude caused by filter are not substantial, it would not affect our results significantly. Thus in this work, we used the filtered data to study the characteristics of Forbush decrease events, although the unfiltered data are also shown in corresponding figures for direct comparison. Further, we repeated the calculations using unfiltered data and find that the final results are not significantly different. There can also be anisotropies intrinsic to the $\mathrm{CME} /$ magnetic cloud itself, arising from a $\boldsymbol{B} \times \nabla N$ drift, where $\boldsymbol{B}$ is the interplanetary magnetic field and $\boldsymbol{\nabla} N$ denotes the cosmic-ray density gradient inside the CME (Bieber \& Evenson 1998; Munakata et al. 2003, 2005; Kuwabara et al. 2004). Such anisotropies can potentially be "mixed" with the diurnal anisotropy, and it is possible that there will still be some residual anisotropy after the filter is applied. This is the case with one of the weaker events that we have studied here; namely 2003 November 20.

To determine the properties of Forbush decrease, we divided the time interval in three parts, the first part before the onset; the second part includes the decrease, while the third part includes the recovery phase. We fit straight lines to the first two parts and an exponential profile for the third part. The point of intersection of the two straight lines defines the onset time for the Forbush decrease, while the intersection of the last two fits determines the minimum of the profile. The difference in the flux between these two points determines the magnitude $M$ of the decrease (Sect. 3), which is measured in term of percentage change in steady flux. The fits for all events used in this work are shown in the respective sections.

In the subsequent analysis we discuss in detail three Forbush decrease events, namely those on 2001 April 11, 2003 October 29, and 2003 November 20, which were associated with $\mathrm{CME} /$ magnetic clouds. The GRAPES-3 data is recorded along nine directions as described earlier. However, in Fig. 8 the data for only the vertical direction is shown for these three events along with measurements from the Tibet neutron monitor at Yangbajing (Muraki et al. 2007) as obtained from http: //cr0. izmiran.rssi.ru/tibt/main.htm. The Tibet neutron monitor has a wide solid-angle of acceptance with the dominant contribution coming from the vertical direction; for that reason, we selected the GRAPES-3 data along the vertical direction for a comparison as shown in Fig. 8. The Tibet neutron monitor was chosen, as Yangbajing $\left(90.5^{\circ} \mathrm{E}\right)$ is located at a similar longitude as Ooty $\left(76.7^{\circ} \mathrm{E}\right)$, and the geomagnetic cutoff rigidities are also not too dissimilar (Ooty $=17 \mathrm{GV}$, and Yangbajing $=14 \mathrm{GV}$ ). The neutron monitor data was scaled down by a factor of 3 to fit the same scale. Here, it needs to be emphasised that both sets of data were subjected to the same low pass filter to remove frequencies higher than $1 \mathrm{day}^{-1}$. The detailed features in the Tibet neutron monitor data follow the GRAPES-3 muon data rather closely for all three Forbush decrease events as seen from Fig. 8.

\section{Proton diffusion into $\mathrm{CME} /$ magnetic cloud}

We are concerned here with the component of the Forbush decrease produced by the difference between the proton density inside and outside the magnetic cloud that intercepts the Earth. To do this, we obtain an estimate of the proton density inside the CME/magnetic cloud produced by the cumulative effect of protons diffusing into the closed magnetic structure of the CME/magnetic cloud as it propagates towards the Earth. We use cross-field diffusion coefficients derived from recent numerical treatments of cosmic ray propagation through turbulent magnetic fields.

Before doing so, it is worth taking a brief look at the typical proton gyro-radius in relation to the overall size of the ejecta to see how far such a picture involving cross-field diffusion would be valid. This is especially pertinent here, since the protons detected by the GRAPES-3 experiment are typically very energetic. The GRAPES-3 experiment can detect muons produced 

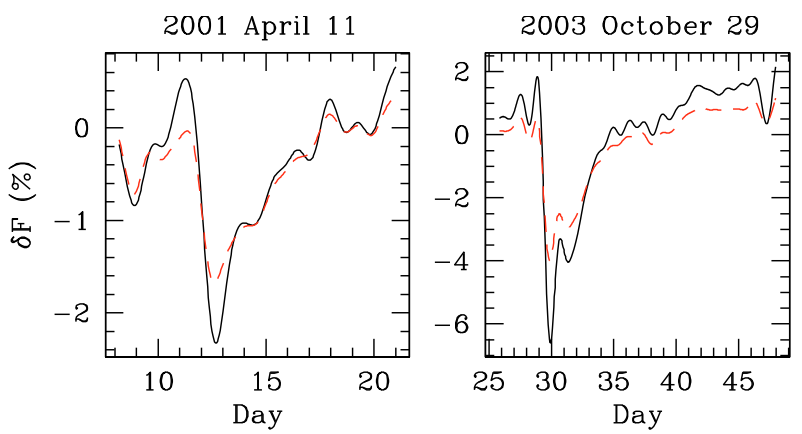

Fig. 8. Data from Tibet neutron monitor (dashed lines) is compared with GRAPES-3 data (solid line) for vertical direction for the 3 events studied in this work. Both the time series are filtered by applying a low pass filter to remove high frequency components. In order to fit in the same scale the neutron monitor data is scaled down by a factor of 3 . by protons of rigidities above $12-42 \mathrm{GV}$, where the rigidity $R g$ is defined by

$R g($ volts $)=\frac{P c}{Z e}=300 B($ Gauss $) r_{\mathrm{L}}(\mathrm{cm})$,

where $P$ is the proton momentum, $c$ the speed of light, $Z$ the charge state (=1 for a proton), $e$ the charge of an electron, $B$ the magnetic field in Gauss, and $r_{\mathrm{L}}$ the proton gyroradius in $\mathrm{cm}$. The magnetic field of a typical near-Earth magnetic cloud is $\sim 10^{-4} \mathrm{G}$, while its radius is $\sim 0.2 \mathrm{AU}$, or $\sim 3 \times 10^{12} \mathrm{~cm}$ (http://lepmfi.gsfc.nasa.gov $/ \mathrm{mfi} / \mathrm{mag}$ cloud_Avg.html). The gyro-radius of a $30 \mathrm{GV}$ proton in the field of a typical near-Earth magnetic cloud is $\sim 10^{12} \mathrm{~cm}$ (from Eq. (1)), or 0.3 times the magnetic cloud radius. Estimates of the magnetic field of a CME near the Sun are notoriously hard to come by, but the rough lower bound of $\sim 0.1 \mathrm{G}$ given by Bastian et al. (2001) can be taken as a working number. The gyro-radius of a $30 \mathrm{GV}$ proton in such a magnetic field is $\sim 3 \times 10^{8} \mathrm{~cm}$, which is around 3 orders of magnitude smaller than the typical size of a CME at such heights, which is a few solar radii (a few times $10^{11} \mathrm{~cm}$ ). Thus the gyro-radius of the typical highenergy proton whose signature is detected by the GRAPES-3 array ranges from $10^{-3}$ to 0.3 times the macroscopic size of the structure into which it penetrates, and the picture of cross-field diffusion is generally valid.

The flux of protons entering the $\mathrm{CME} /$ magnetic cloud at a given time is

$F\left(\mathrm{~cm}^{-2} \mathrm{~s}^{-1}\right)=D_{\perp} \frac{\partial N_{\mathrm{a}}}{\partial r}$,

where $F$ is in units of number per $\mathrm{cm}^{2}$ per sec, $D_{\perp}$ is the cross-field diffusion coefficient, and $N_{\mathrm{a}}$ the ambient density of high-energy protons. This diffusion takes place throughout the cross-section of the expanding CME, so the total number of high-energy protons that will have diffused into the CME after a time $T$ is

$U_{\mathrm{i}}=\int_{0}^{T} A(t) F(t) \mathrm{d} t=\int_{0}^{T} D_{\perp} A(t) \frac{\partial N_{\mathrm{a}}}{\partial r} \mathrm{~d} t$,

where $A(t)$ is the cross-sectional area of the CME at time $t$. The integration extends from the time the CME is first observed in the LASCO field of view $(t=0)$ through the time $(t=T)$ when it arrives at the Earth as a magnetic cloud. A reasonable estimate for $\partial N_{\mathrm{a}} / \partial r$ would be

$\frac{\partial N_{\mathrm{a}}}{\partial r} \simeq \frac{N_{\mathrm{a}}}{R(t)}$

where $R(t)$ is the CME cross-sectional radius at time $t$. Assuming the CME to be an expanding "flux-rope" whose length increases with time as its cross-sectional area expands (see http:// lepmfi.gsfc.nasa.gov/mfi/Mag_Cloud_Model.html for a cartoon), we can use

$A(t)=2 \pi R(t) L(t)$,

where $L(t)$ is the length of the flux-rope cylinder at time $t$, and can be related to the "height" $H$ of the CME above the solar limb via

$L(t)=2 \pi H(t)$.

The height above the solar limb $H$ is the most commonly measured quantity for limb CMEs, but it is not clear how well it can be estimated for the halo CMEs we are considering. We consider this aspect further in Sect. 4 below. Using Eqs. (5), (6), and (4) in Eq. (3), we have

$U_{\mathrm{i}}=4 \pi^{2} N_{\mathrm{a}} \int_{0}^{T} D_{\perp} H(t) \mathrm{d} t$.

The density inside the near-Earth magnetic cloud would be

$N_{\mathrm{i}}=\frac{U_{\mathrm{i}}}{\pi R(T)^{2} L(T)}$

and it can be related to the magnitude $M$ of the Forbush decrease via

$\alpha M=\frac{N_{\mathrm{a}}-N_{\mathrm{i}}}{N_{\mathrm{a}}}=\frac{\Delta N}{N_{\mathrm{a}}}=1-\frac{4 \pi \int_{0}^{T} D_{\perp} H(t) \mathrm{d} t}{R^{2}(T) L(T)}$,

where $L(T)$ and $R(T)$ denote the length and cross-sectional radius at time $T$, when the ejecta has reached the Earth. The quantity $\alpha$ denotes the fraction of the total decrease that can be attributed to the CME/magnetic cloud. As mentioned earlier, this fraction is typically around 50\% (Wibberenz et al. 1997), so we adopt $\alpha=0.5$ in our calculations. We discuss the effect of variations in $\alpha$ on our final result in Sect. 7.

\subsection{Cross-field diffusion coefficient}

We now turn our attention to the cross-field diffusion coefficient $D_{\perp}$, which governs the diffusion of the ambient highenergy protons into the CME across the magnetic fields that enclose it. Protons can travel perpendicular to the direction of the average magnetic field as a result of normal transport of particles due to scattering or drift, as well as the braiding/mixing of the magnetic field itself. The extent of cross-field diffusion depends on several parameters, such as the proton rigidity (which indicates how tightly the proton is bound to the magnetic field) 
Table 1. Derived parameters for Forbush decrease for the 2001 April 11 event.

\begin{tabular}{llllllllll}
\hline \hline Quantity & NW & N & NE & W & V & E & SW & S & SE \\
\hline FD magnitude & 2.51 & 2.51 & 1.87 & 2.92 & 2.71 & 2.04 & 2.58 & 2.37 & 1.79 \\
FD onset & $15: 36$ & $17: 31$ & $19: 26$ & $10: 05$ & $11: 45$ & $16: 19$ & $06: 58$ & $09: 22$ & $12: 45$ \\
FD end $^{1}$ & $15: 07$ & $13: 55$ & $12: 43$ & $12: 57$ & $12: 43$ & $11: 45$ & $09: 36$ & $11: 02$ & $11: 17$ \\
$\sigma^{2}$ (Kolmogorov) & 0.02 & 0.02 & 0.02 & 0.02 & 0.02 & 0.02 & 0.02 & 0.02 & 0.02 \\
\hline
\end{tabular}

${ }^{1}$ The FD end times refer to 2001 April 12.

and the level of turbulence, which can contribute to field line transport, as well as proton scattering.

The issue of the cross-field diffusion coefficient is a subject of intensive ongoing research, and a good way to proceed for our purposes is to use the convenient parametrisation of the results of extensive Monte Carlo simulations of cosmic rays propagating through tangled magnetic fields given by Candia \& Roulet (2004). Their results can be regarded as a superset of similar work (e.g., Giacalone \& Jokipii 1999; Casse et al. 2002); it contains all the preceding results and also extends the parameter regime of the simulations. They give the following fit for the "parallel" diffusion coefficient $D_{\|}$(which is due to scattering of the particles back and forth along the mean field, as the field is subject to random turbulent fluctuations):

$D_{\|}=c L_{\max } \rho \frac{N_{\|}}{\sigma^{2}} \sqrt{\left(\frac{\rho}{\rho_{\|}}\right)^{2(1-\gamma)}+\left(\frac{\rho}{\rho_{\|}}\right)^{2}}$,

where $c$ is the speed of light and the quantities $N_{\|}, \gamma$ and $\rho_{\|}$ are constants specific to different kinds of turbulence whose values are given in Table 1 of Candia \& Roulet (2004). The quantity $L_{\max }$ is the maximum length scale of the turbulence. Matthaeus et al. (2005) quote a value of $L_{\max }=10^{6} \mathrm{~km}$, based on multi-spacecraft measurements of solar wind turbulence near the Earth. This is similar to values quoted by other authors (e.g., Manoharan et al. 1994) who use very different methods. We take the quantity $L_{\max }$ to be a fixed number from near the Sun to the Earth. Candia \& Roulet (2004) call $\rho$ the "rigidity", but it is somewhat different from our usual definition. It is related to the usual rigidity $R g$ by

$\rho=\frac{r_{\mathrm{L}}}{L_{\max }}=\frac{R g}{B_{0} L_{\max }}$,

where $B_{0}$ is the mean magnetic field. The quantity $\sigma^{2}$ is the turbulence level, and is defined as

$\sigma^{2} \equiv \frac{\left\langle B_{r}^{2}\right\rangle}{B_{0}^{2}}$

where $B_{r}$ is the turbulent magnetic field and the angular braces denote an ensemble average. The cross-field diffusion coefficient $\left(D_{\perp}\right)$ is related to the parallel one $\left(D_{\|}\right)$by

$\frac{D_{\perp}}{D_{\|}}= \begin{cases}N_{\perp}\left(\sigma^{2}\right)^{a_{\perp}}, & \rho \leq 0.2 \\ N_{\perp}\left(\sigma^{2}\right)^{a_{\perp}}\left(\frac{\rho}{0.2}\right)^{-2}, & \rho>0.2 .\end{cases}$

The quantities $N_{\perp}$ and $a_{\perp}$ are constants specific to different kinds of turbulent spectra, and are given in Table 1 of Candia \& Roulet (2004). For concreteness, we assume a Kolmogorov turbulence spectrum in our calculations. Equations (10) and (13) jointly define the cross-field diffusion coefficient. The magnetic field $B_{0}$ of the CME changes (weakens) as it propagates from the Sun to the Earth, which means that $\rho$ varies with time. We do not know how $B_{0}$ varies with heliocentric distance, but we can assume that the flux is frozen into the CME as it propagates from the Sun to the Earth. It may be argued that some of the magnetic flux will be dissipated in driving the CME. However, on average, even the flux detected by near-Earth magnetic clouds is enough to account for some $74 \%$ of what is required to drive the CME from the Sun to the Earth (Subramanian \& Vourlidas 2007). The frozen-flux assumption is therefore fairly accurate. This means that the product of the magnetic field and the cross-sectional area of the CME remains approximately constant, i.e.,

$B_{0}(t)=B_{\mathrm{MC}}\left[\frac{R(T)}{R(t)}\right]^{2}$

where $B_{\mathrm{MC}}$ is the magnetic field inside the magnetic cloud. The quantity $R(t)$ is related to the measured expansion velocity $V_{\exp }$ and the starting radius $R_{0}$ of the halo CME by

$R(t)=V_{\exp } t+R_{0}$

We note that the measured quantities $R_{0}$ and $R(t)$ are the lateral extents of the halo CME measured in the plane of the sky, and are thus representative of the lateral expansion of the CME crosssection.

\subsection{Constraining $\sigma^{2}$}

For a given Forbush decrease event, we measure the quantity $M$, and Eqs. (15), (14), (13), (11), and (10) jointly define the cross-field diffusion coefficient $D_{\perp}$, which can be used in Eq. (9). Unfortunately, there is not much simplification possible in the expression for $D_{\perp}$; the quantity $\rho$ typically straddles regimes such that both the terms under the square root in Eq. (10) and both the branches of Eq. (13) need to be retained, and when $D_{\perp}$ is finally substituted into Eq. (9) the integral needs to be evaluated numerically. As we see below, we have concrete observational values for all the quantities used in Eq. (9) except the turbulence level $\sigma^{2}$ (Eq. (12)), and it is this quantity that we seek to constrain. Owing to the different cutoff rigidities in different bins and remaining anisotropies, the magnitude $M$ of the Forbush decrease (for a given event) differs from bin to bin. Consequently, we obtain slightly different estimates for $\sigma^{2}$ for a given event, which can be regarded as a scatter (Tables 1-3). For a given bin, we adopt a single number for the proton rigidity, which is equal to the cutoff rigidity for that bin. For instance, we adopt $R g=17 \mathrm{GV}$ for the vertical bin, and the values of $R g$ for the other bins correspond to the respective cutoff rigidities listed in Sect. 2.1. We note that this is a simplifying assumption, for a muon detected in a given bin can in fact be produced by protons possessing a range of energies. We next describe the characteristics of the three events we have selected for our study. 
Table 2. Derived parameters for Forbush decrease for 2003 October 29 event.

\begin{tabular}{llllllllll}
\hline \hline Quantity & NW & N & NE & W & V & E & SW & S & SE \\
\hline FD magnitude & 6.81 & 6.78 & 5.21 & 7.76 & 7.76 & 5.85 & 6.92 & 6.82 & 5.26 \\
FD onset & $00: 58$ & $23: 46^{*}$ & $22: 34^{*}$ & $00: 58$ & $00: 00$ & $23: 17^{*}$ & $00: 53$ & $00: 14$ & $23: 31^{*}$ \\
FD end & $18: 00$ & $17: 16$ & $16: 19$ & $19: 12$ & $18: 14$ & $16: 19$ & $18: 43$ & $17: 16$ & $15: 07$ \\
$\sigma^{2}$ (Kolmogorov) & 2.48 & 2.49 & 2.55 & 2.45 & 2.45 & 2.52 & 2.48 & 2.49 & 2.55 \\
\hline
\end{tabular}

* These times refer to 2003 October 28.

Table 3. Derived parameters for Forbush decrease for 2003 November 20 event.

\begin{tabular}{llllllllll}
\hline \hline Quantity & $\mathrm{NW}$ & $\mathrm{N}$ & $\mathrm{NE}$ & $\mathrm{W}$ & $\mathrm{V}$ & $\mathrm{E}$ & $\mathrm{SW}$ & $\mathrm{S}$ & $\mathrm{SE}$ \\
\hline FD magnitude & 0.90 & 0.91 & 0.89 & 1.26 & 1.24 & 1.04 & 1.24 & 1.24 & 0.88 \\
FD onset & $13: 12^{*}$ & $11: 46^{*}$ & $02: 10^{*}$ & $01: 26$ & $05: 02$ & $21: 07$ & $06: 29$ & $11: 02$ & $21: 07$ \\
FD end $^{1}$ & $04: 57$ & $04: 26$ & $02: 32$ & $06: 00$ & $04: 54$ & $05: 00$ & $04: 16$ & $04: 12$ & $02: 54$ \\
$\sigma^{2}$ (Kolmogorov) & 0.06 & 0.06 & 0.06 & 0.06 & 0.06 & 0.06 & 0.06 & 0.06 & 0.06 \\
\hline
\end{tabular}

* These times refer to 2003 November 21.

${ }^{1}$ The times for FD end refer to 2003 November 24.

\section{2001 April 11 event}

\subsection{Magnetic cloud}

This event had its genesis in a halo CME that started at 05:30 UT on 2001 April 10 (http://cdaw.gsfc.nasa.gov/ CME_list/). The starting radius was $R_{0}=2.84 R_{\odot}$, and the lateral (plane-of-sky) expansion speed was $V_{\exp }=2411 \mathrm{~km} \mathrm{~s}^{-1}$. Based on an examination of several halo CMEs, Schwenn et al. (2005) conclude that the radial speed $V$ is typically 0.88 times the expansion speed $V_{\exp }$ of a halo CME. This gives $V=$ $0.88 V_{\exp }=2121 \mathrm{~km} \mathrm{~s}^{-1}$. The corresponding magnetic cloud was detected by the ACE spacecraft at 04:00 UT on 2001 April 12, while the shock reached the Earth at 15:18 UT on 2001 April 11 (Lynch et al. 2003). Munakata et al. (2003, 2005) have modelled the magnetic cloud associated with this event as a cylinder with a Gaussian cross-sectional profile for the cosmic-ray density depletion inside it. They analysed the cosmic-ray directional anisotropy from muon detector measurements from several stations. Assuming that this anisotropy arises from a diamagnetic drift due to the interplanetary magnetic field and the density gradient inside the magnetic cloud, they have derived best-fit parameters for the 3D cylindrical model of the magnetic cloud as it passes across the Earth. The length of the flux rope at time $t$ is

$L(t)=2 \pi H(t)=2 \pi\left(V t+R_{0}\right)$.

The length $L$ at time $T$ is simply

$L(T)=2 \pi 1 \mathrm{AU}$.

It may be noted that we are using the value $R_{0}$ for the initial value of the observed height $H$ above the solar limb. This is not quite right for full halo CMEs, since $R_{0}$ is really the observed lateral extent of the CME. However, this is the only concrete observational quantity available, and the error is not expected to contribute significantly to our final result for $\sigma^{2}$ (see Sect. 7). The time elapsed between the first observation of the halo CME and the detection of the magnetic cloud by the ACE spacecraft is $46.5 \mathrm{~h}$. The average speed of the magnetic cloud by the time it reaches the ACE spacecraft is $640.6 \mathrm{~km} \mathrm{~s}^{-1}$ (Lynch et al. 2003), and the spacecraft is located around $1.5 \times 10^{6} \mathrm{~km}$ from the Earth. We therefore estimate that the magnetic cloud would have taken $\sim 0.6 \mathrm{~h}$ to traverse the distance between ACE and the Earth. The total time $T=46.5+0.6=47.1 \mathrm{~h}$. The radius of the magnetic cloud is $R(T)=0.106 \mathrm{AU}$, and magnetic field at the centre of the cloud is estimated to be (Lynch et al. 2003) $B_{\mathrm{MC}}=17.8 \mathrm{nT}$.

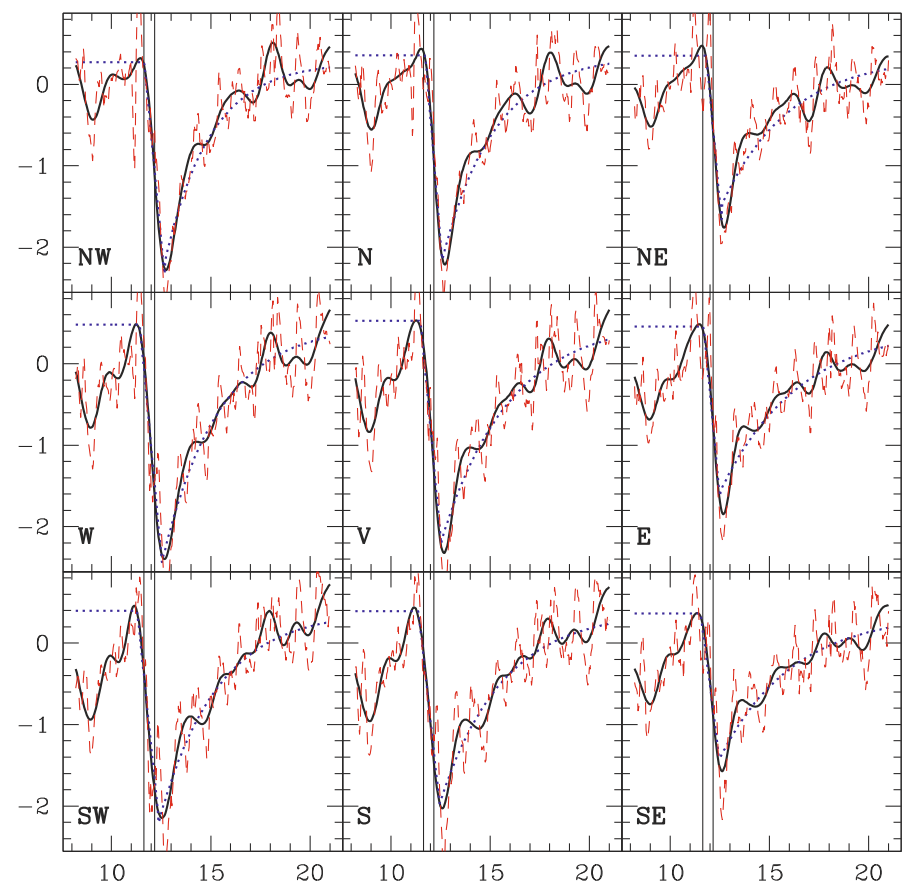

Fig. 9. The muon flux in the nine directions is shown for the Forbush decrease on 2001 April 11. The fluxes are shown as percentage deviation from mean values. The dashed red lines show the unfiltered data, while solid black lines show the data after applying a low-pass filter. The dotted blue lines show the fits to filtered data. The vertical lines in each panel mark the arrival times of shock and magnetic cloud at the Earth.

\subsection{Forbush decrease}

Table 1 gives the start and end times and magnitudes of the Forbush decrease in different bins for this event, as well as the computed values of the turbulence level $\sigma^{2}$ (Eq. (12)). The shock associated with this event impacted the Earth at 15:18 UT, 2001 April 11 (Huttunen et al. 2005) and the magnetic cloud first intercepted the ACE spacecraft at 04:00 UT, 2001 April 12 (Lynch et al. 2003). Evidently, the magnetic cloud was observed on the Earth after the start of the Forbush decrease and well before its end in all the directional bins. It therefore stands to reason that it will have contributed substantially to the overall Forbush decrease. As discussed in Sect. 3, we adopt a value of $\alpha=0.5$, 


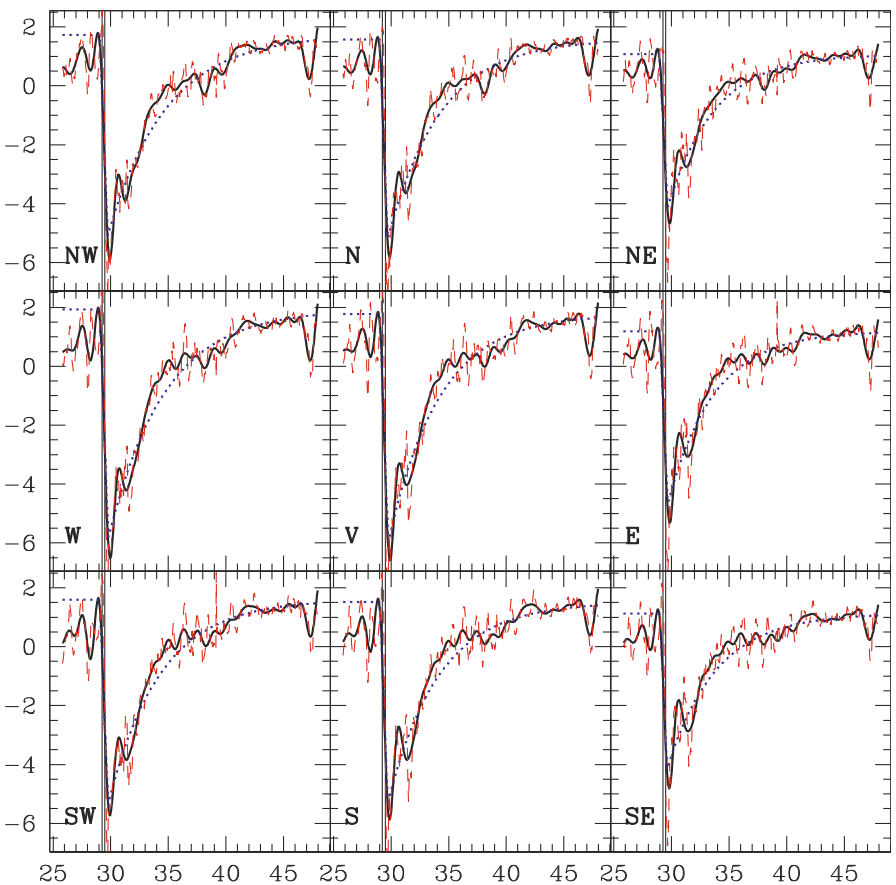

Fig. 10. The muon flux in each of the nine directions for the Forbush decrease on 2003 October 29. The line-styles and markings are the same as Fig. 9.

which means that the magnetic cloud contributes to $50 \%$ of the overall Forbush decrease.

\section{2003 October 29 event}

\subsection{Magnetic cloud}

This was an exceptional event associated with an X17 flare close to the central meridian, together with a very fast halo CME. This full halo CME was first observed at a starting radius of $R_{0}=$ $5.84 R_{\odot}$ at 11:30 UT on 2003 October 28 (http : / / cdaw . gsfc. nasa.gov/CME_list/). As with the other events, the quantity $R_{0}$ really represents the first observed lateral extent of the halo CME. The quantity $L(T)$ can be taken to be the same as defined in Eq. (17). The lateral expansion speed of the CME as discerned from coronograph data is $V_{\exp }=2459 \mathrm{~km} \mathrm{~s}^{-1}$, and the radial expansion speed is related to $V_{\exp }$ by $V=0.88 V_{\exp }$ as before. The length of the flux rope at a given time $t$ is given as before by Eq. (16).

Kuwabara et al. (2004) have derived best-fit parameters for the $3 \mathrm{D}$ cylindrical model of the magnetic cloud as it passes across the Earth using the methods described in Munakata et al. $(2003,2005)$. They estimate the radius of the magnetic cloud to be $R(T)=0.14 \mathrm{AU}$, the magnetic field at the centre of the cloud to be $B_{\mathrm{MC}}=44 \mathrm{nT}$, and the time of closest approach of the cloud axis to be 16:27 UT on 2003 October 29. The ACE observations (Huttunen et al. 2005) reveal that the magnetic cloud made its entry at 12:00 UT, 2003 October 29. The time elapsed between the first observation of the halo CME (11:30 UT, 2003 October 28) and the time it was intercepted at the ACE spacecraft (12:00 UT, 2003 October 29) is $24.5 \mathrm{~h}$.

We could not find data for the magnetic cloud speed as measured by the ACE spacecraft. We therefore used the estimate of $\sim 1400 \mathrm{~km} \mathrm{~s}^{-1}$ from Kuwabara et al. (2004) for the magnetic cloud speed. At this speed, the magnetic cloud would take only $\sim 0.3 \mathrm{~h}$ to traverse the distance of $1.5 \times 10^{6} \mathrm{~km}$ between the

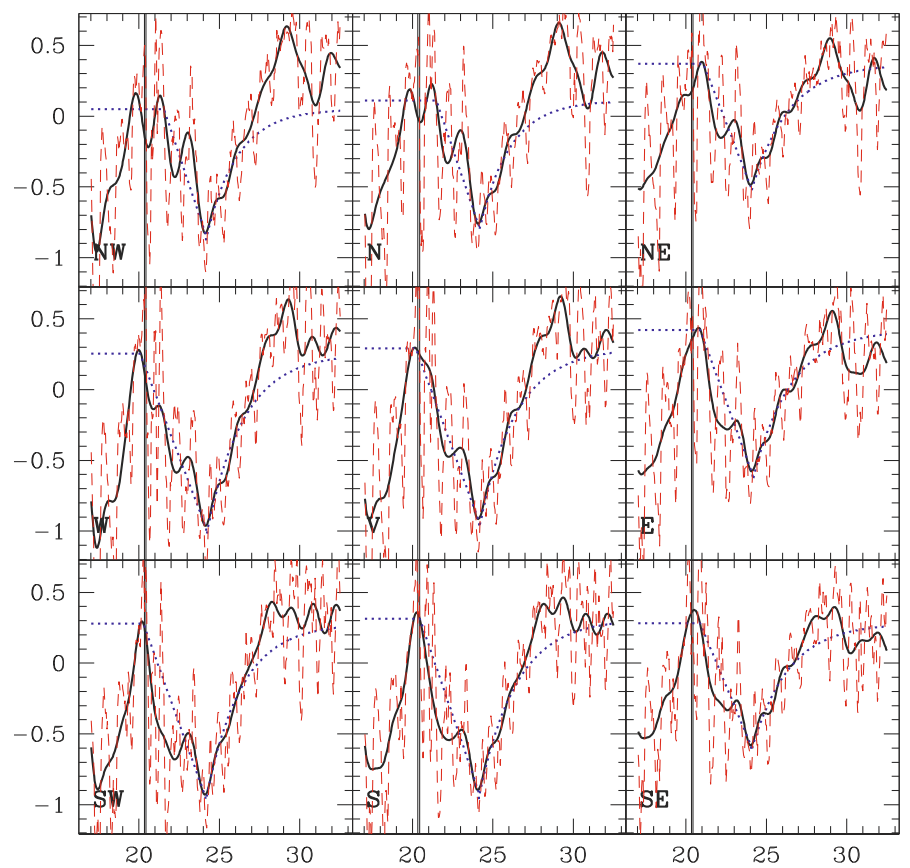

Fig. 11. The muon flux in each of the nine directions is shown for the Forbush decrease on 2003 November 20. The linestyles and markings are the same as those for Fig. 9.

ACE spacecraft and the Earth. The total time $T$ is therefore $T \simeq 24.8 \mathrm{~h}$. It may be noted that the CME liftoff speeds for this event and one previously discussed (2001 April 11) are similar. However, the event of 2001 April 11 decelerates significantly en route to the Earth, as a result of which the total travel time $T$ is almost twice as long as for this event.

\subsection{Forbush decrease}

The Forbush decrease on 2003 October 29 was among the largest observed by the GRAPES-3 muon telescope, and it is described in detail in Nonaka et al. (2006). The time profiles of the decrease as observed by the different modules is shown in Fig. 10 of that paper. The derived parameters from the Forbush decrease profiles in the different directional bins are given in Table 2, where the magnitude of Forbush decrease derived here from the fit to the filtered data are systematically lower than those observed in the raw data, which were reported earlier (Nonaka et al. 2006). The onset and recovery times in the 2003 October 29 FD event were relatively faster and thus the reduction in magnitude is largely caused by the removal of a higher frequency component due to the use of a low-pass filter to remove the diurnal variations. The shock ahead of the ejecta impacted the Earth at 05:58 UT, 2003 October 29 (Dryer et al. 2004), and the magnetic cloud was detected by the ACE spacecraft at 12:00 UT, 2003 October 29. We noted earlier that it takes around $0.3 \mathrm{~h}$ for the cloud to travel the distance between ACE and the Earth at a speed of $1400 \mathrm{~km} \mathrm{~s}^{-1}$; in addition to this, if one accounts for the travel time between the leading edge of the cloud and the centre using the same speed (and a cloud radius of $0.14 \mathrm{AU}$ ), it results in an additional time of $4 \mathrm{~h}$ and $21 \mathrm{~min}$. When added to $12: 00 \mathrm{UT}$, the final result agrees very well with the intercept time of 16:27 UT quoted by Kuwabara et al. (2004) for the centre of the cloud. As with the 2001 April 11 event, the magnetic cloud intercepts the Earth after the start of the Forbush decrease and well before its end in all the directional bins. The density 
Table 4. Summary of various observationally derived parameters for the three events we consider, leading to $\sigma^{2}$ (Eq. (12)) as our final result.

\begin{tabular}{llllllc}
\hline \hline Event & $\begin{array}{l}R_{0} \\
\left(R_{\odot}\right)\end{array}$ & $\begin{array}{l}V_{\text {exp }} \\
\left(\mathrm{km} \mathrm{s}^{-1}\right)\end{array}$ & $\begin{array}{l}T \\
(\mathrm{~h})\end{array}$ & $\begin{array}{l}R(T) \\
(\mathrm{AU})\end{array}$ & $\begin{array}{l}B_{\mathrm{MC}} \\
(\mathrm{nT})\end{array}$ & Average $\sigma^{2}$ (Kolmogorov) \\
\hline 2001 April 11 & 2.84 & 2411 & 47.1 & 0.10 & 17.8 & $2 \%$ \\
2003 October 29 & 5.84 & 2459 & 24.8 & 0.14 & 44 & $249 \%$ \\
2003 November 20 & 6.3 & 1660 & 49.4 & 0.12 & 50 & $6 \%$ \\
\hline
\end{tabular}

depletion inside the magnetic cloud will therefore account for a substantial part of the overall Forbush decrease. This justifies our use of $\alpha=0.5$ (Sect. 3) to account for the fraction of the overall Forbush decrease related to the CME/magnetic cloud.

\section{2003 November 20 event}

\subsection{Magnetic cloud data}

This magnetic cloud event was associated with an erupting filament and a halo CME, and was observed very well (Wang et al. 2006; Huttunen et al. 2005) by the WIND and ACE spacecraft. Wang et al. (2006) have modelled this cloud as a cylindrical, force-free flux rope to determine its radius and other parameters. As we shall see, the Forbush decrease associated with this event is less than those for the other two events studied in this paper. However, it illustrates the diversity of events observed with the GRAPES-3 experiment, and is associated with the best-studied magnetic cloud of the three events considered in this paper.

The radius of the magnetic cloud as measured by the ACE spacecraft is (Wang et al. 2006) $R(T)=T(7 \mathrm{~h}) \times V_{\max }=$ $7 \times 3600 \times\left(750 \mathrm{~km} \mathrm{~s}^{-1}\right)=1.89 \times 10^{7} \mathrm{~km}$, where we have assumed that magnetic cloud moves with the maximum in-situ speed of the ambient solar wind behind the shock $V_{\max }=750 \mathrm{~km} \mathrm{~s}^{-1}$. The magnetic field at the centre of the cloud is estimated to be (Wang et al. 2006) $B_{\mathrm{MC}}=50 \mathrm{nT}$. The most likely solar origin of this magnetic cloud is a full halo CME that was first recorded at 08:50 UT on 2003 November 18 at a height of $R_{0}=$ $6.3 R_{\odot}=2.76 \times 10^{12} \mathrm{~cm}$ (http://cdaw.gsfc.nasa.gov/ CME_list/). The plane-of-sky expansion speed $V_{\exp }$ of the halo $\mathrm{CME}$ as recorded in the CME catalogue is $V_{\exp }=1660 \mathrm{~km} \mathrm{~s}^{-1}$. Following Schwenn et al. (2005), we computed the radial speed $V=0.88 V_{\exp }=1460 \mathrm{~km} \mathrm{~s}^{-1}$. The total time elapsed between 08:50 UT, 2003 November 18 (the time the CME was observed at $6.3 R_{\odot}$ ) and $10.1 \mathrm{UT}, 2003$ November 20 (the time the mag cloud was intercepted by ACE, Wang et al. 2006) is $1.76 \times 10^{5} \mathrm{~s}$. Since the ACE satellite is at a distance of $\sim 1.5 \times 10^{6} \mathrm{~km}$ from the Earth, we estimate that it takes $\sim 2000 \mathrm{~s}$ for the magnetic cloud to arrive at the Earth at the solar wind velocity $V_{\max }=750 \mathrm{~km} \mathrm{~s}^{-1}$. Therefore, $T=1.76 \times 10^{5}+2000=1.78 \times 10^{5} \mathrm{~s}$.

\subsection{Forbush decrease data}

Table 3 gives the data derived from the Forbush decrease observed on 2003 November 20. This is a comparatively smaller event, and there is considerable variation in start times for the different bins. This variation is most probably due to uncertainty caused by oscillations in the fluxes before the decrease (mainly in the NE and $\mathrm{N}$ bins), some of which even remain in the filtered data. In this case since the magnitude of Forbush decrease is comparatively small, the diurnal variations are comparable to the decrease, and it is not possible to fit the unfiltered data to obtain any meaningful estimates for the parameters of the Forbush decrease; hence, it is necessary to only use the filtered data for this purpose. Furthermore, it is very likely that there is some CME-related anisotropy remaining in the data, and removing it requires the technique used by Munakata et al. $(2003,2005)$ and Kuwabara et al. (2004), which is outside the scope of the current paper.

The shock driven by the CME arrived at the ACE spacecraft at 07:27 UT (Huttunen et al. 2005). Since the average shock speed from the sun to the Earth is $1020 \mathrm{~km} \mathrm{~s}^{-1}$ (Wang et al. 2006), it would take around $1470 \mathrm{~s}$ to traverse the $\sim 1.5 \mathrm{mil}-$ lion $\mathrm{km}$ between the ACE satellite and the Earth, and it would have reached the Earth at around 07:51 UT, 2003 November 20. On the other hand, the ACE spacecraft first intercepts the magnetic cloud at 10:06 UT (Wang et al. 2006), and the cloud would have reached the Earth at around 10:36 UT on 2003 November 20, taking some $2000 \mathrm{~s}$ to traverse the distance between ACE and the Earth at the solar wind velocity of $V_{\max }=750 \mathrm{~km} \mathrm{~s}^{-1}$. Comparing these times with the start times of the Forbush decrease given in Table 3, it is evident that the magnetic cloud start time is before the start time in most of the bins, and after it in a few of them. However, the end of the Forbush decrease takes place well after the entry of the magnetic cloud. Thus the overall decrease should have contributions from both the shock and the ejecta/magnetic cloud, and we assume that the CME/magnetic cloud-related component of the decrease accounts for $50 \%$ of the total decrease, as discussed earlier in Sect. 3. The estimate of the turbulence level $\sigma^{2}$ (Eq. (12)) given in Table 3 was computed according to the procedure discussed in Sect. 3.

\section{Results}

We use the observational parameters for each event summarised in Tables 1-3 using the procedure outlined in Sect. 3.2 to constrain the turbulence level $\sigma^{2}$ (Eq. (12)) for each event. This quantity represents the ratio of the energy density in the turbulent magnetic fields to that in the mean magnetic field. We computed $\sigma^{2}$ separately for each directional bins, and the results are listed in the last row of Tables 1-3. The average value of $\sigma^{2}$ for each event is quoted in Table 4.

We note that the turbulence levels near the CME/ejecta front for the 2001 April 11 and 2003 November 20 events are fairly small (around 2\% and 6\% respectively). These numbers are commensurate with what would be expected in the general quiescent solar wind at a few tens of $R_{\odot}$ (e.g., Spangler 2002). The turbulence level near the CME/ejecta front of 2003 October 29, on the other hand, is significantly higher (around 249\%) and is representative of fairly strong turbulence. This is not surprising, since this event was a much stronger one, and was associated with a significantly faster shock. It is conceivable that strong turbulence associated with the shock front can affect the vicinity of the CME/ejecta behind it.

Our final result regarding the turbulence level $\sigma^{2}$ is not very sensitive to the precise value of $\alpha$ (Eq. (9)). A change of a factor 
of 2 in $\alpha$ changes $\sigma^{2}$ by 2 to $4 \%$. We also pointed out earlier that the estimate for the initial height of the full halo CMEs is made from the first observation of the lateral extent of the CME, $R_{0}$. This is the only concrete observable quantity for halo CMEs, and we have verified that an error of $1 R_{\odot}$ in $R_{0}$ will result in an error of around $2 \%$ in the estimate of the turbulence level $\sigma^{2}$. The decrease magnitudes vary somewhat from one direction to the next, as noted earlier. We have verified that these differences in the estimate of the Forbush decrease magnitude contribute a scatter of only around $2 \%$ to our final result for the turbulence level $\sigma^{2}$. The parameters that influence our final result most strongly are the average propagation speed $V$ and the total time $T$ elapsed between the time when the CME was first observed and when it was intercepted as a magnetic cloud by the ACE spacecraft.

\section{Summary}

We have focused on the contribution of the CME/ejecta in producing Forbush decreases observable from the Earth, using data for three events observed with the GRAPES-3 tracking muon telescope at Ooty. We selected events that clearly have their origins in full-halo CMEs that originate close to the centre of the solar disc. The Forbush decreases produced by these events will therefore be due to the shock, as well as to the CME/ejecta. While it is somewhat difficult to precisely separate out the shockassociated and CME/ejecta-associated contributions, it is reasonable to assume that the CME/ejecta effects accounts for $50 \%$ of the overall Forbush decrease. We used CME measurements from LASCO and magnetic cloud measurements from WIND and ACE to obtain various geometrical parameters of the CME and its near-Earth manifestation. The CME is a substantially closed magnetic structure that starts out with a negligible number of high-energy protons inside it. We consider the diffusion of high-energy cosmic-ray protons into the CME as it propagates and expands on its way from the Sun to the Earth. Since the diffusion primarily takes place across the magnetic fields that enclose the CME, we use an expression from the literature for the cross-field diffusion coefficient that is derived from extensive simulations of cosmic rays propagating through turbulent magnetic fields. The diffusion coefficient depends upon several quantities like the ratio of the proton Larmor radius to the maximum scale length of the turbulence $\rho$ (Eq. (11)) and the level of turbulence $\sigma^{2}$ (Eq. (12)). The turbulence near the CME is expected to result in a magnetic-field line random walk, leading to scattering of protons and contributing to their cross-field diffusion.

We constrained the magnitude of $\sigma^{2}$ (which is the ratio of the energy density in the random magnetic fields to that in the largescale magnetic field) in the vicinity of the CME front for each of the events we analysed. We find that the turbulence level $\sigma^{2}$ near the CME front for the 2001 April 11 Forbush decrease event is $\sim 2 \%$ and $\sim 6 \%$ for the event of 2003 November 20, while it is $\sim 249 \%$ for the much more energetic event of 2003 October 29. These estimates may be regarded as an average over the journey of the CME from the Sun to the Earth, for we do not take its possible radial evolution into account. The radial evolution of $\sigma^{2}$ is unlikely to be appreciable, at least in the outer corona, as we discuss below. If we take turbulent magnetic field fluctuations to be representative of those in the electron density (Spangler 2002), we note that the power-law index for electron density fluctuations $\Delta N_{\mathrm{e}}$ as a function of heliocentric distance ranges from - 2.2 (Manoharan 1993) to -1.7 (Fallows et al. 2002). This is fairly close to the power law index of the background electron density $N_{\mathrm{e}}$, which is around -2 in the outer corona (e.g.,
Leblanc et al. 1998). In other words, the quantity $\Delta N_{\mathrm{e}} / N_{\mathrm{e}}$ does not vary appreciably with distance from the Sun, and we can surmise that $\sigma^{2}$ behaves similarly.

To the best of our knowledge, these are the first quantitative estimates of the turbulence levels near CME fronts. Manoharan et al. (2000) have noted that the turbulent density spectra of the plasma near CMEs are significantly flatter than what is observed in the slow and fast solar wind, and are similar to what exists in the near-Sun solar wind acceleration region. However, they were unable to comment on the level of the turbulence. Badruddin (2002) suggests that the level of turbulence near the $\mathrm{CME} /$ magnetic cloud might influence the magnitude of the Forbush decrease. However, he only considered the local effect of the turbulence, while our treatment takes into account the cumulative effect of the turbulence near the CME on the crossfield diffusion of protons into it. Wibberenz et al. (1998) quote the results of Vanhoefer (1996) who makes a simple estimate of the cross-field diffusion coefficient by calculating the "filling in" timescale of the CME by energetic protons. This method results in a rather low estimate for the diffusion coefficient, and they speculate that this might be due to the smooth magnetic fields enclosing the CME. Our treatment considers this aspect in detail, incorporating the expansion of the CME, as well as results for the cross-field diffusion coefficient adopted from extensive numerical simulations.

Acknowledgements. We thank D. B. Arjunan, A. Jain, the late S. Karthikeyan, K. Manjunath, S. Murugapandian, S. D. Morris, B. Rajesh, B. S. Rao, C. Ravindran, K. C. Ravindran, and R. Sureshkumar for their help in the testing, installation, and operating the proportional counters and the associated electronics and during data acquisition. We acknowledge the administrative services of V. Viswanathan. We thank A. A. Basha, G. P. Francis, I. M. Haroon, V. Jeyakumar, and K. Ramadass for their help in the fabrication, assembly, and installation of various mechanical components and detectors. The Japanese members of the GRAPES-3 collaboration acknowledge the partial financial support from the Ministry of Education and Science of the Government of Japan for the GRAPES3 experiment. Part of this work was carried out when P. Subramanian was at the Indian Institute of Astrophysics. We are grateful to the Tibet neutron monitor group for the use of their data. We thank the referee, M. Duldig, for several comments and suggestions that have significantly improved the content of the paper. This paper is dedicated to the memory of S. Karthikeyan who passed away recently.

\section{References}

Badruddin 2002, Ap\&SS, 281, 651

Badruddin, Yadav, R. S., \& Yadav, N. R. 1986, Sol. Phys., 105, 413

Badruddin, Venkatesan, D., \& Zhu, B. Y. 1991, Sol. Phys., 134, 203

Bastian, T. S., Pick, M., Kerdraon, A., Maia, D., \& Vourlidas, A. 2001, ApJ, 558, L65

Bieber, J. W., \& Evenson, P. 1998, Geophys. Res. Lett., 25, 2955

Bothmer, V., \& Schwenn, R. 1998, Ann. Geophysicae, 16, 1

Bothmer, V., Desai, M. I., Marsden, R. G., et al. 1996, A\&A, 316, 493

Bothmer, V., et al. 1997, Proc. 25th Intl. Cosmic Ray Conf., 1, 333

Burlaga, L. F., Sittler, E., Mariani, F., \& Schwenn, R. 1981, J. Geophys. Res., 86,6673

Candia, J., \& Roulet, E. 2004, J. Cosmol. Astropart. Phys., 10, 007

Cane, H. V. 2000, Space Sci. Rev., 93, 55

Cane, H. V., \& Richardson, I. G. 1997, Proc. 25th Intl. Cosmic Ray Conf., 1, 397

Cane, H. V., Richardson, I. G., von Rosenvinge, T. T., \& Wibberenz, G. 1994, J. Geophys. Res., 99, 21429

Cane, H. V., Richardson, I. G., \& Wibberenz, G. 1995, Proc. 24th Intl. Cosmic Ray Conf., 4, 377

Cane, H. V., Richardson, I. G., \& Wibberenz, G. 1997, J. Geophys. Res., 102, 7075

Casse, F., Lemoine, M., \& Pelletier, G. 2002, Phys. Rev. D, 65, 023002

Chih, P. C., \& Lee, M. A. 1986, J. Geophys. Res., 91, 2903

Cooke, D. J., Humble, J. E., Shea, M. A., et al. 1991, Il Nuovo Cimento C, 14, 213 
Dryer, M., et al. 2004, Space Weather, 2, S09001, doi : 10.1029/2004SW000087

Fallows, R. A., Williams, P. J. S., \& Breen, A. R. 2002, Annales Geophysicae, 20, 1279

Forsyth, R. J., Bothmer, V., Cid, C., et al. 2006, Space Sci. Rev., 123, 383

Giacalone, J., \& Jokipii, J. R. 1999, ApJ, 520, 204

Gupta, S. K., Aikawa, Y., Gopalakrishnan, N. V., et al. 2005, Nucl. Instrum. Methods A, 540, 311

Hayashi, Y., Aikawa, Y., Gopalakrishnan, N. V., et al. 2005, Nucl. Instrum. Methods A, 545, 643

Huttunen, K. E. J., Schwenn, R., Bothmer, V., \& Koskinen, H. E. J. 2005, Annales Geophysicae, 23, 625

Kuwabara, T., et al. 2004, Geophys. Res. Lett., 31, L19803

Leblanc, Y., Dulk, G. A., \& Bougeret, J.-L. 1998, Sol. Phys., 183, 165

le Roux, J. A., \& Potgieter, M. S. 1991, A\&A, 243, 531

Lockwood, J. A., Webber, W. R., \& Debrunner, H. 1991, J. Geophys. Res., 96, 11587

Lynch, B. J., Zurbuchen, T. H., Fisk, L. A., \& Antiochos, S. K. 2003, J. Geophys. Res., 108, 1239

Manoharan, P. K. 1993, Sol. Phys., 148, 153

Manoharan, P. K., Kojima, M., \& Misawa, H. 1994, J. Geophys. Res., 99, 23411

Manoharan, P. K., Kojima, M., Gopalswamy, N., Kondo, T., \& Smith, Z. 2000 ApJ, 530, 1061
Matthaeus, W. H., et al. 2005, Phys. Rev. Lett., 95, 231101

Munakata, K., Kuwabara, T., Bieber, J. W., et al. 2003, Proc. 28th Int. Cosmic Ray Conf., 6, 3561

Munakata, K., Kuwabara, T., Bieber, J. W., et al. 2005, Adv. Space Res., 36, 2357

Muraki, Y., Tsuchiya, H., Fujiki, K., et al. 2007, Astropart. Phys., 28, 119

Nishida, A. 1983, J. Geophys. Res., 88, 785

Nonaka, T., et al. 2006, Phys. Rev. D, 74, 052003

Sanderson, T. R. T., Beeck, J., Marsden, G. R., et al. 1990, Proc. 21st Int. Cosmic Ray Conf., 6, 251

Schwenn, R., Dal Lago, A., Huttunen, E., \& Gonzalez, W. D. 2005, Annales Geophysicae, 23, 1033

Spangler, S. R. 2002, ApJ, 576, 997

Subramanian, P., \& Vourlidas, A. 2007, A\&A, 467, 685

Vanhoefer, O. 1996, Master's thesis, University of Kiel

Venkatesan, D., \& Badruddin 1990, Space Sci. Rev., 52, 121

Wang, Y., Zhou, G., Ye, P., Wang, S., \& Wang, J. 2006, ApJ, 651, 1245

Wibberenz, G., Cane. H. V., \& Richardson, I. G. 1997, Proc. 25th Intl. Cosmic Ray Conf., 1, 397

Wibberenz, G., le Roux, J. A., Potgieter, M. S., \& Bieber, J. W. 1998, Space Sci. Rev., 83, 309

Wimmer-Schweingruber, R. F., et al. 2006, Space Sci. Rev., 123, 177

Zhang, G., \& Burlaga, L. F. 1988, J. Geophys. Res., 93, 2511 